\title{
Exponential Stabilization of a Class of Time-Varying Delay Systems with Nonlinear Perturbations
}

\author{
Yazhou Tian, ${ }^{1,2}$ Yuanli Cai, ${ }^{1}$ Yuangong Sun, ${ }^{3}$ and Tongxing $\mathrm{Li}^{2}$ \\ ${ }^{1}$ School of Electronic and Information Engineering, Xian Jiaotong University, Xi'an, Shaanxi 710049, China \\ ${ }^{2}$ Qingdao Technological University, Feixian, Shandong 273400, China \\ ${ }^{3}$ School of Mathematical Sciences, University of Jinan, Jinan, Shandong 250022, China \\ Correspondence should be addressed to Yuanli Cai; ylicai@mail.xjtu.edu.cn and Tongxing Li; litongx2007@163.com
}

Received 3 April 2015; Accepted 25 June 2015

Academic Editor: Xinguang Zhang

Copyright (C) 2015 Yazhou Tian et al. This is an open access article distributed under the Creative Commons Attribution License, which permits unrestricted use, distribution, and reproduction in any medium, provided the original work is properly cited.

\begin{abstract}
This paper addresses the problem of exponential stabilization of a class of time-varying delay systems with nonlinear perturbations. These perturbations are related not only with current state $x(t)$ and the delayed state $x(t-h(t))$ but also with $\beta(t)$, where $\beta(t)$ is a continuous function defined on $[0,+\infty)$. With the delay interval divided into two equidistant subintervals, a novel Lyapunov functional is introduced, and several new exponential stabilization criteria are derived in terms of linear matrix inequalities (LMIs) by employing reciprocally convex approach. Two examples are given to illustrate the effectiveness of the main results.
\end{abstract}

\section{Introduction}

Time delay is commonly encountered in various physical and engineering systems such as aircraft, biological systems, and networked control systems. Since the existence of time delays causes poor performance, oscillation, or even instability, it is very important to investigate stability analysis for systems with time delays before designing control systems. On the other hand, the systems almost present some uncertainties because it is not easy to obtain an exact mathematical model due to environmental noise, uncertain or slowly varying parameters, and so forth. Therefore, considerable amounts of efforts have been done to the stability and stabilization of time-delay systems and time-delay systems with nonlinear perturbations; see, for example, [1-25] and the references cited therein.

Recently, Zhang et al. [12] considered interval time-varying delay systems and obtained some delay-dependent conditions by employing Finsler's lemma. Combining the descriptor model transformation and the integral inequality method, Han [3] investigated the robust stability of linear systems with time-varying delay and nonlinear perturbations and obtained several improved stability conditions. On the basis of free weighting matrices technique, robust stabilization criteria for neutral systems with nonlinear perturbations were reported in [9]. Wang et al. [6] introduced a new parameter in the Lyapunov functional for the timevarying delay systems with nonlinear perturbations and obtained less conservative results, whereas the range of the time delays considered in the paper was assumed from zero to an upper bound. Note that the stability investigated in the above-mentioned papers was primarily focused on asymptotic stability. Using delay decomposition method and Finsler's lemma, Liu et al. [24] studied the exponential stability of neutral systems with interval time-varying delays and nonlinear perturbations. So far, there are few articles concerning the problem of exponential stabilization of timevarying delay systems with nonlinear perturbations. Thuan et al. [16] provided a detailed analysis for the problem of designing state feedback controllers to exponential stabilization of time-delay systems with nonlinear perturbations by using the integral inequality method and constructing a Lyapunov functional containing the triple integral terms. However, there still exists a gap for reducing both the conservatism and the number of decision variables. 
In this paper, we study the exponential stabilization of a class of time-delay systems with nonlinear perturbations. The main contributions of this paper can be summarized as follows: (i) a novel Lyapunov functional containing the center point of time-delay interval is constructed; (ii) compared with the systems studied in $[3,8,16]$, the nonlinear perturbations of (1) are related not only with the current state $x(t)$ and the delayed state $x(t-h(t))$ but also with $\beta(t)$, where $\beta(t)$ is a continuous function satisfying $\int_{0}^{+\infty} \beta^{2}(s) e^{2 \alpha s} d s<+\infty$ and $\alpha$ is a positive constant; new sufficient conditions are obtained that ensure the stability of a closed-loop system, which extend and improve the main results of [16]. Furthermore, the stabilization conditions are shown to be less conservative than those reported in Zhang et al. [12] when there are no nonlinear perturbations in the system. Finally, two numerical examples are presented to demonstrate the effectiveness and advantages of the main results.

Notation. Throughout the paper, $R^{n}$ denotes the $n$-dimensional Euclidean space with vector norm $\|\cdot\|$, and $R^{n \times m}$ is the set of all $n \times m$-dimensional real matrices. $I$ denotes the identity matrix of appropriate dimensions, and the superscript " $T$ " stands for matrix transposition. The notation $P>$ $0(\geq 0)$ means that $P$ is symmetric and positive (semipositive) definite. $\lambda_{\min }(A)$ and $\lambda_{\max }(A)$ denote the minimum and maximum eigenvalues of $A$, respectively. In addition, in symmetric block matrices or long matrix expressions, we use an asterisk $(*)$ to represent a term that is induced by symmetry.

\section{Problem Description and Preliminaries}

Consider the following system with a nonlinear perturbation:

$$
\begin{aligned}
\dot{x}(t)= & A x(t)+D x(t-h(t)) \\
& +f(t, x(t), x(t-h(t)))+B u(t), \quad t \geq 0, \\
x(t)= & \phi(t), \quad t \in\left[-h_{2}, 0\right],
\end{aligned}
$$

where $x(t) \in R^{n}$ is the state vector, $u(t) \in R^{m}$ is the control input vector, $A, D \in R^{n \times n}$, and $B \in R^{n \times m}, \phi(t) \in$ $C\left(\left[-h_{2}, 0\right], R^{n}\right)$ with $\|\phi\|=\sup _{t \in\left[-h_{2}, 0\right]}\{\|\phi(t)\|,\|\dot{\phi}(t)\|\}$, where $C\left(\left[-h_{2}, 0\right], R^{n}\right)$ is the Banach space of continuous functions. The delay $h(t)$ is time-varying and satisfies

$$
0 \leq h_{1} \leq h(t) \leq h_{2}, \quad \dot{h}(t) \leq \mu
$$

where $\mu$ is a positive constant and $h_{1}$ and $h_{2}$ are constants representing the lower and upper bounds of the delay, respectively. $f(t, x(t), x(t-h(t)))$ is a nonlinear perturbation satisfying

$$
\begin{aligned}
& \|f(t, x(t), x(t-h(t)))\| \\
& \quad \leq a\|x(t)\|+b\|x(t-h(t))\|+\beta(t),
\end{aligned}
$$

where $a$ and $b$ are positive scalars and $\beta(t)$ satisfies $\int_{0}^{+\infty} \beta^{2}(s) e^{2 \alpha s} d s<+\infty$.
Remark 1 . In $[3,8,16]$, the authors assumed that the nonlinear terms satisfy

$$
\begin{gathered}
f^{T}(t, x(t)) f(t, x(t)) \leq a^{2} x^{T}(t) F^{T} F x(t), \\
g^{T}(t, x(t-h(t))) g(t, x(t-h(t))) \\
\quad \leq b^{2} x^{T}(t-h(t)) G^{T} G x(t-h(t)),
\end{gathered}
$$

where $F$ and $G$ are constant matrices and $a$ and $b$ are positive scalars. It is obvious that the assumptions on the nonlinear terms given in (2) and (3) are more general.

The following definitions and lemmas will be used for providing the main results in the sequel.

Definition 2. Given a scalar $\alpha>0$ : system (1) with $u(t)=0$ is $\alpha$-stable if there exists a positive number $\gamma>0$ such that every solution $x(t, \phi)$ of the system satisfies

$$
\|x(t, \phi)\| \leq \gamma e^{-\alpha t}, \quad t \geq 0 .
$$

Definition 3. Given a scalar $\alpha>0$ : system (1) is $\alpha$-stabilizable if there exists a state feedback control $u(t)=K x(t)$ such that the closed-loop system

$$
\begin{aligned}
\dot{x}(t)= & (A+B K) x(t)+D x(t-h(t)) \\
& +f(t, x(t), x(t-h(t))), \quad t \geq 0, \\
x(t)= & \phi(t), \quad t \in\left[-h_{2}, 0\right]
\end{aligned}
$$

is $\alpha$-stable.

Lemma 4 (see [25]). For any $x, y \in R^{n}$ and a positive symmetric definite matrix $N \in R^{n \times n}$,

$$
\pm 2 y^{T} x \leq x^{T} N^{-1} x+y^{T} N y \text {. }
$$

Lemma 5 (lower bound lemma for reciprocal convexity; see [20]). Let $f_{1}, f_{2}, \ldots, f_{N}: R^{m} \rightarrow R$ have positive values in an open subset $D$ of $R^{m}$. Then, the reciprocally convex combination of $f_{i}$ over $D$ satisfies

$$
\min _{\left\{\alpha_{i} \mid \alpha_{i}>0, \sum_{i} \alpha_{i}=1\right\}} \sum_{i} \frac{1}{\alpha_{i}} f_{i}(t)=\sum_{i} f_{i}(t)+\max _{g_{i, j}(t)} \sum_{i \neq j} g_{i, j}(t)
$$

subject to

$$
\begin{aligned}
& \left\{g_{i, j}: R^{m} \longrightarrow R, g_{j, i}(t) \triangleq g_{i, j}(t),\left[\begin{array}{cc}
f_{i}(t) & g_{i, j}(t) \\
g_{i, j}(t) & f_{j}(t)
\end{array}\right]\right. \\
& \quad \geq 0\} .
\end{aligned}
$$




\section{Main Results}

We use the following notation for the convenience:

$$
\begin{aligned}
\bar{h}= & \frac{h_{1}+h_{2}}{2}, \\
\delta= & \frac{h_{2}-h_{1}}{2}, \\
\lambda= & \lambda_{\min }\left(P^{-1}\right), \\
\Gamma= & \lambda_{\max }\left(P^{-1}\right)+h_{1} \lambda_{\max }\left(P^{-1} Q_{1} P^{-1}\right)+\left(h_{2}-h_{1}\right) \lambda_{\max }\left(P^{-1} Q_{2} P^{-1}\right)+\left(h_{2}-h_{1}\right) \lambda_{\max }\left[\begin{array}{cc}
P^{-1} R_{11} P^{-1} & P^{-1} R_{12} P^{-1} \\
* & P^{-1} R_{22} P^{-1}
\end{array}\right] \\
& +\frac{\left(3 h_{1}+h_{2}\right)\left(h_{2}-h_{1}\right)^{2}}{16} \lambda_{\max }\left(P^{-1} S_{1} P^{-1}\right)+\frac{\left(h_{1}+3 h_{2}\right)\left(h_{2}-h_{1}\right)^{2}}{16} \lambda_{\max }\left(P^{-1} S_{2} P^{-1}\right)+\frac{h_{1}^{3}}{2} \lambda_{\max }\left(P^{-1} S_{3} P^{-1}\right), \\
\Lambda= & \Gamma\|\phi\|^{2}+6 \int_{0}^{+\infty} \beta^{2}(s) e^{2 \alpha s} d s, \\
\xi^{T}(t)= & {\left[x^{T}(t) x^{T}(t-h(t)) x^{T}\left(t-h_{1}\right) x^{T}(t-\bar{h}) x^{T}\left(t-h_{2}\right) \dot{x}^{T}(t)\right] . }
\end{aligned}
$$

The following theorem presents an exponential stabilization condition for (1).

Theorem 6. Let $\alpha>0$ and assume that conditions (2) and (3) are satisfied. If there exist matrices $P>0, Q_{1}>0, Q_{2}>0$, $S_{i}>0(i=1,2,3),\left[\begin{array}{cc}R_{11} & R_{12} \\ * & R_{22}\end{array}\right]>0, M_{1}, M_{2}$, and $Y$ such that the following LMIs hold

$\Sigma_{1}$

$$
\left[\begin{array}{cccccccc}
\Sigma_{11} & D P & \Sigma_{13} & 0 & 0 & P A^{T}+Y^{T} B^{T} & a^{2} P & 0 \\
* & \Sigma_{22} & \Sigma_{23} & \Sigma_{24} & 0 & P D^{T} & 0 & b^{2} P \\
* & * & \Sigma_{33} & \Sigma_{34} & 0 & 0 & 0 & 0 \\
* & * & * & \Sigma_{44} & \Sigma_{45} & 0 & 0 & 0 \\
* & * & * & * & \Sigma_{55} & 0 & 0 & 0 \\
* & * & * & * & * & \Sigma_{66} & 0 & 0 \\
* & * & * & * & * & * & -\frac{1}{6} a^{2} I & 0 \\
* & * & * & * & * & * & 0 & -\frac{1}{6} b^{2} I
\end{array}\right]
$$$$
<0 \text {, }
$$

$\Sigma_{2}$

$$
=\left[\begin{array}{cccccccc}
\Sigma_{11} & D P & \Sigma_{13} & 0 & 0 & P A^{T}+Y^{T} B^{T} & a^{2} P & 0 \\
* & \bar{\Sigma}_{22} & 0 & \bar{\Sigma}_{24} & \bar{\Sigma}_{25} & P D^{T} & 0 & b^{2} P \\
* & * & \Sigma_{33} & \bar{\Sigma}_{34} & 0 & 0 & 0 & 0 \\
* & * & * & \Sigma_{44} & \bar{\Sigma}_{45} & 0 & 0 & 0 \\
* & * & * & * & \Sigma_{55} & 0 & 0 & 0 \\
* & * & * & * & * & \Sigma_{66} & 0 & 0 \\
* & * & * & * & * & * & -\frac{1}{6} a^{2} I & 0 \\
* & * & * & * & * & * & 0 & -\frac{1}{6} b^{2} I
\end{array}\right]
$$

$$
\left[\begin{array}{cc}
S_{i} & M_{i} \\
* & S_{i}
\end{array}\right] \geq 0 \quad(i=1,2),
$$

where

$$
\begin{aligned}
\Sigma_{11}= & A P+P A^{T}+B Y+Y^{T} B^{T}+2 \alpha P+I+Q_{1} \\
& -e^{-2 \alpha h_{1}} S_{3}, \\
\Sigma_{22}= & (1-\mu) e^{-2 \alpha h_{2}} Q_{2}+e^{-2 \alpha \bar{h}} M_{1}+e^{-2 \alpha \bar{h}} M_{1}^{T} \\
& -2 e^{-2 \alpha \bar{h}} S_{1}, \\
\Sigma_{33}= & e^{-2 \alpha h_{1}} Q_{2}+e^{-2 \alpha h_{1}} R_{11}-e^{-2 \alpha h_{1}} Q_{1}-e^{-2 \alpha \bar{h}} S_{1} \\
& -e^{-2 \alpha h_{1}} S_{3}, \\
\Sigma_{44}= & e^{-2 \alpha h_{1}} R_{22}-e^{-2 \alpha \bar{h}} R_{11}-e^{-2 \alpha \bar{h}} S_{1}-e^{-2 \alpha h_{2}} S_{2}, \\
\Sigma_{55}= & -e^{-2 \alpha \bar{h}} R_{22}-e^{-2 \alpha h_{2}} S_{2}, \\
\Sigma_{66}= & h_{1}^{2} S_{3}+\delta^{2} S_{2}+\delta^{2} S_{1}+I-2 P, \\
\Sigma_{13}= & e^{-2 \alpha h_{1}} S_{3}, \\
\Sigma_{23}= & e^{-2 \alpha \bar{h}}\left(S_{1}-M_{1}\right), \\
\Sigma_{24}= & e^{-2 \alpha \bar{h}}\left(S_{1}-M_{1}^{T}\right) \\
\Sigma_{34}= & e^{-2 \alpha h_{1}} R_{12}+e^{-2 \alpha \bar{h}} M_{1}^{T}, \\
\Sigma_{45}= & -e^{-2 \alpha \bar{h}} R_{12}+e^{-2 \alpha h_{2}} S_{2}, \\
\Sigma_{22}= & -(1-\mu) e^{-2 \alpha h_{2}} Q_{2}+e^{-2 \alpha h_{2}} M_{2}+e^{-2 \alpha h_{2}} M_{2}^{T} \\
& -2 \alpha h_{2} S_{2}, \\
& \\
& \\
&
\end{aligned}
$$




$$
\begin{aligned}
& \bar{\Sigma}_{24}=e^{-2 \alpha h_{2}}\left(S_{2}-M_{2}\right), \\
& \bar{\Sigma}_{25}=e^{-2 \alpha h_{2}}\left(S_{2}-M_{2}^{T}\right), \\
& \bar{\Sigma}_{34}=e^{-2 \alpha h_{1}} R_{12}+e^{-2 \alpha \bar{h}} S_{1}, \\
& \bar{\Sigma}_{45}=-e^{-2 \alpha \bar{h}} R_{12}+e^{-2 \alpha h_{2}} M_{2}^{T},
\end{aligned}
$$

then system (1) is robustly $\alpha$-stabilizable, the state feedback control $u(t)=Y P^{-1} x(t)$, and the solution $x(t, \phi)$ of the closedloop system satisfies

$$
\|x(t, \phi)\| \leq \sqrt{\frac{\Lambda}{\lambda}} e^{-\alpha t}, \quad t \geq 0 .
$$

Proof. Let us denote

$$
\begin{aligned}
\bar{Q}_{i} & =P^{-1} Q_{i} P^{-1}, \\
\bar{M}_{i} & =P^{-1} M_{i} P^{-1}, \quad i=1,2 ; \\
\bar{S}_{i} & =P^{-1} S_{i} P^{-1}, \quad i=1,2,3 ; \\
\bar{R}_{11} & =P^{-1} R_{11} P^{-1}, \\
\bar{R}_{12} & =P^{-1} R_{12} P^{-1}, \\
\bar{R}_{22} & =P^{-1} R_{22} P^{-1} .
\end{aligned}
$$

Define a Lyapunov functional by

$$
V\left(x_{t}\right)=V_{1}\left(x_{t}\right)+V_{2}\left(x_{t}\right)+V_{3}\left(x_{t}\right) \text {, }
$$

where

$$
\begin{aligned}
& V_{1}\left(x_{t}\right)=x^{T}(t) P^{-1} x(t), \\
& V_{2}\left(x_{t}\right)=\int_{t-h_{1}}^{t} e^{2 \alpha(s-t)} x^{T}(s) \bar{Q}_{1} x(s) d s \\
& +\int_{t-h(t)}^{t-h_{1}} e^{2 \alpha(s-t)} x^{T}(s) \bar{Q}_{2} x(s) d s \\
& +\int_{t-\bar{h}}^{t-h_{1}} e^{2 \alpha(s-t)}\left[\begin{array}{c}
x(s) \\
x(s-\delta)
\end{array}\right]^{T} \\
& \quad\left[\begin{array}{c}
\bar{R}_{11} \bar{R}_{12} \\
* \\
*
\end{array} \bar{R}_{22}\right]\left[\begin{array}{c}
x(s) \\
x(s-\delta)
\end{array}\right] d s, \\
& V_{3}\left(x_{t}\right)=\delta \int_{-\bar{h}}^{-h_{1}} \int_{t+s}^{t} e^{2 \alpha(\tau-t)} \dot{x}^{T}(\tau) \bar{S}_{1} \dot{x}(\tau) d \tau d s \\
& +\delta \int_{-h_{2}}^{-\bar{h}} \int_{t+s}^{t} e^{2 \alpha(\tau-t)} \dot{x}^{T}(\tau) \bar{S}_{2} \dot{x}(\tau) d \tau d s \\
& +h_{1} \int_{-h_{1}}^{0} \int_{t+s}^{t} e^{2 \alpha(\tau-t)} \dot{x}^{T}(\tau) \bar{S}_{3} \dot{x}(\tau) d \tau d s .
\end{aligned}
$$

Calculating the time derivative of $V\left(x_{t}\right)$ along the trajectories of (6), we conclude that

$$
\begin{aligned}
& \dot{V}_{1}\left(x_{t}\right) \\
& =x^{T}(t)\left[P^{-1}(A+B K)+(A+B K)^{T} P^{-1}\right] x(t) \\
& +2 x^{T}(t) P^{-1} D x(t-h(t)) \\
& +2 x^{T}(t) P^{-1} f(t, x(t), x(t-h(t))), \\
& \dot{V}_{2}\left(x_{t}\right) \\
& \leq-2 \alpha V_{2}\left(x_{t}\right)+x^{T}(t) \bar{Q}_{1} x(t) \\
& -(1-\mu) e^{-2 \alpha h_{2}} x^{T}(t-h(t)) \bar{Q}_{2} x(t-h(t)) \\
& +e^{-2 \alpha h_{1}} x^{T}\left(t-h_{1}\right)\left[\bar{Q}_{2}-\bar{Q}_{1}\right] x\left(t-h_{1}\right) \\
& +e^{-2 \alpha h_{1}}\left[\begin{array}{c}
x\left(t-h_{1}\right) \\
x(t-\bar{h})
\end{array}\right]^{T}\left[\begin{array}{cc}
\bar{R}_{11} & \bar{R}_{12} \\
* & \bar{R}_{22}
\end{array}\right]\left[\begin{array}{c}
x\left(t-h_{1}\right) \\
x(t-\bar{h})
\end{array}\right] \\
& -e^{-2 \alpha \bar{h}}\left[\begin{array}{c}
x(t-\bar{h}) \\
x\left(t-h_{2}\right)
\end{array}\right]^{T}\left[\begin{array}{ll}
\bar{R}_{11} & \bar{R}_{12} \\
* & \bar{R}_{22}
\end{array}\right]\left[\begin{array}{c}
x(t-\bar{h}) \\
x\left(t-h_{2}\right)
\end{array}\right] \text {, } \\
& \dot{V}_{3}\left(x_{t}\right) \\
& \leq-2 \alpha V_{3}\left(x_{t}\right)+\dot{x}^{T}(t)\left(h_{1}^{2} \bar{S}_{3}+\delta^{2} \bar{S}_{2}+\delta^{2} \bar{S}_{1}\right) \dot{x}(t) \\
& -\delta e^{-2 \alpha h_{2}} \int_{t-h_{2}}^{t-\bar{h}} \dot{x}^{T}(s) \bar{S}_{2} \dot{x}(s) d s \\
& -\delta e^{-2 \alpha \bar{h}} \int_{t-\bar{h}}^{t-h_{1}} \dot{x}^{T}(s) \bar{S}_{1} \dot{x}(s) d s \\
& -h_{1} e^{-2 \alpha h_{1}} \int_{t-h_{1}}^{t} \dot{x}^{T}(s) \bar{S}_{3} \dot{x}(s) d s .
\end{aligned}
$$

By virtue of Lemma 4, we have

$$
\begin{aligned}
& 2 x^{T}(t) P^{-1} f(t, x(t), x(t-h(t))) \\
& \quad \leq x^{T}(t) P^{-1} P^{-1} x(t) \\
& \quad+f^{T}(t, x(t), x(t-h(t))) f(t, x(t), x(t-h(t))) \\
& \quad=x^{T}(t) P^{-1} P^{-1} x(t)+\|f(t, x(t), x(t-h(t)))\|^{2} .
\end{aligned}
$$

Using (3) and the inequality $(H+J+L)^{2} \leq 3 H^{2}+3 J^{2}+3 L^{2}$, where $H, J$, and $L$ are constants, we obtain

$$
\begin{aligned}
& \|f(t, x(t), x(t-h(t)))\|^{2} \\
& \quad \leq(a\|x(t)\|+b\|x(t-h(t))\|+\beta(t))^{2} \\
& \quad \leq 3 a^{2}\|x(t)\|^{2}+3 b^{2}\|x(t-h(t))\|^{2}+3 \beta^{2}(t) .
\end{aligned}
$$


It follows from (22) and (23) that

$$
\begin{aligned}
2 x^{T}(t) & P^{-1} f(t, x(t), x(t-h(t))) \\
\leq & x^{T}(t)\left(P^{-1} P^{-1}+3 a^{2} I\right) x(t) \\
& +3 b^{2} x^{T}(t-h(t)) x(t-h(t))+3 \beta^{2}(t) .
\end{aligned}
$$

Combining (19) and (24), we get

$$
\begin{aligned}
& \dot{V}_{1}\left(x_{t}\right) \leq-2 \alpha V_{1}\left(x_{t}\right)+x^{T}(t)\left[P^{-1}(A+B K)\right. \\
& \left.\quad+(A+B K)^{T} P^{-1}+2 \alpha P^{-1}+P^{-1} P^{-1}+3 a^{2} I\right] x(t) \\
& \quad+2 x^{T}(t) P^{-1} D x(t-h(t))+3 b^{2} x^{T}(t-h(t)) x(t \\
& \quad-h(t))+3 \beta^{2}(t) .
\end{aligned}
$$

Now, we estimate the upper bounds of the last three integral terms in inequality (21) as follows.

(i) Assume first that $h_{1} \leq h(t) \leq \bar{h}$. From Jensen's inequality [19] and Lemma 5,

$$
\begin{aligned}
& -\delta \int_{t-\bar{h}}^{t-h_{1}} \dot{x}^{T}(s) \bar{S}_{1} \dot{x}(s) d s \\
& =-\delta \int_{t-\bar{h}}^{t-h(t)} \dot{x}^{T}(s) \bar{S}_{1} \dot{x}(s) d s \\
& -\delta \int_{t-h(t)}^{t-h_{1}} \dot{x}^{T}(s) \bar{S}_{1} \dot{x}(s) d s \\
& \leq-\frac{\delta}{\bar{h}-h(t)}\left(\int_{t-\bar{h}}^{t-h(t)} \dot{x}(s) d s\right)^{T} \\
& \cdot \bar{S}_{1}\left(\int_{t-\bar{h}}^{t-h(t)} \dot{x}(s) d s\right) \\
& -\frac{\delta}{h(t)-h_{1}}\left(\int_{t-h(t)}^{t-h_{1}} \dot{x}(s) d s\right)^{T} \\
& \cdot \bar{S}_{1}\left(\int_{t-h(t)}^{t-h_{1}} \dot{x}(s) d s\right) \\
& \leq-\left[\begin{array}{c}
x(t-h(t))-x(t-\bar{h}) \\
x\left(t-h_{1}\right)-x(t-h(t))
\end{array}\right]^{T} \\
& \cdot\left[\begin{array}{cc}
\bar{S}_{1} & \bar{M}_{1} \\
* & \bar{S}_{1}
\end{array}\right]\left[\begin{array}{c}
x(t-h(t))-x(t-\bar{h}) \\
x\left(t-h_{1}\right)-x(t-h(t))
\end{array}\right],
\end{aligned}
$$

and so

$$
\begin{aligned}
& -\delta e^{-2 \alpha \bar{h}} \int_{t-\bar{h}}^{t-h_{1}} \dot{x}^{T}(s) \bar{S}_{1} \dot{x}(s) d s \\
& \leq-\left[\begin{array}{c}
x(t-h(t))-x(t-\bar{h}) \\
x\left(t-h_{1}\right)-x(t-h(t))
\end{array}\right]^{T} \\
& .\left[\begin{array}{cc}
e^{-2 \alpha \bar{h}} \bar{S}_{1} & e^{-2 \alpha \bar{h}} \bar{M}_{1} \\
* & e^{-2 \alpha \bar{h}} \bar{S}_{1}
\end{array}\right]\left[\begin{array}{c}
x(t-h(t))-x(t-\bar{h}) \\
x\left(t-h_{1}\right)-x(t-h(t))
\end{array}\right] .
\end{aligned}
$$

On the other hand, if $h(t)=\bar{h}$ or $h(t)=h_{1}$, then $\int_{t-\bar{h}}^{t-h(t)} \dot{x}(s) d s=0$ or $\int_{t-h(t)}^{t-h_{1}} \dot{x}(s) d s=0$, respectively. Hence, inequality (27) holds.

Using Jensen's inequality [19], it is not difficult to arrive at the inequalities

$$
\begin{gathered}
-h_{1} e^{-2 \alpha h_{1}} \int_{t-h_{1}}^{t} \dot{x}^{T}(s) \bar{S}_{3} \dot{x}(s) d s \\
\leq-\left[x(t)-x\left(t-h_{1}\right)\right]^{T} \\
\cdot e^{-2 \alpha h_{1}} \bar{S}_{3}\left[x(t)-x\left(t-h_{1}\right)\right], \\
-\delta e^{-2 \alpha h_{2}} \int_{t-h_{2}}^{t-\bar{h}} \dot{x}^{T}(s) \bar{S}_{2} \dot{x}(s) d s \\
\leq-\left[x(t-\bar{h})-x\left(t-h_{2}\right)\right]^{T} \\
\cdot e^{-2 \alpha h_{2}} \bar{S}_{2}\left[x(t-\bar{h})-x\left(t-h_{2}\right)\right] .
\end{gathered}
$$

It follows now from (6) that

$$
\begin{gathered}
2 \dot{x}^{T}(t) P^{-1}[(A+B K) x(t)+D x(t-h(t)) \\
+f(t, x(t), x(t-h(t)))-\dot{x}(t)]=0 .
\end{gathered}
$$

On the other hand,

$$
\begin{aligned}
& 2 \dot{x}^{T}(t) P^{-1} f(t, x(t), x(t-h(t))) \\
& \leq \dot{x}^{T}(t) P^{-1} P^{-1} \dot{x}(t) \\
&+f^{T}(t, x(t), x(t-h(t))) f(t, x(t), x(t-h(t))) \\
&= \dot{x}^{T}(t) P^{-1} P^{-1} \dot{x}(t)+\|f(t, x(t), x(t-h(t)))\|^{2} \\
& \leq \dot{x}^{T}(t) P^{-1} P^{-1} \dot{x}(t)+3 a^{2} x^{T}(t) x(t) \\
&+3 b^{2} x^{T}(t-h(t)) x(t-h(t))+3 \beta^{2}(t) .
\end{aligned}
$$

Therefore, formulas (19)-(31) imply that

$$
\dot{V}\left(x_{t}\right)+2 \alpha V\left(x_{t}\right) \leq \xi^{T}(t) \Phi_{1} \xi(t)+6 \beta^{2}(t),
$$

where

$\Phi_{1}$

$$
=\left[\begin{array}{cccccc}
\Phi_{11} & P^{-1} D & \Phi_{13} & 0 & 0 & (A+B K)^{T} P^{-1} \\
* & \Phi_{22} & \Phi_{23} & \Phi_{24} & 0 & D^{T} P^{-1} \\
* & * & \Phi_{33} & \Phi_{34} & 0 & 0 \\
* & * & * & \Phi_{44} & \Phi_{45} & 0 \\
* & * & * & * & \Phi_{55} & 0 \\
* & * & * & * & * & \Phi_{66}
\end{array}\right],
$$




$$
\begin{aligned}
\Phi_{11} & \\
= & P^{-1}(A+B K)+(A+B K)^{T} P^{-1}+2 \alpha P^{-1} \\
& +P^{-1} P^{-1}+\bar{Q}_{1}-e^{-2 \alpha h_{1}} \bar{S}_{3}+6 a^{2} I, \\
\Phi_{22} & \\
= & -(1-\mu) e^{-2 \alpha h_{2}} \bar{Q}_{2}+e^{-2 \alpha \bar{h}} \bar{M}_{1}+e^{-2 \alpha \bar{h}} \bar{M}_{1}^{T} \\
& -2 e^{-2 \alpha \bar{h}} \bar{S}_{1}+6 b^{2} I, \\
\Phi_{33} & \\
= & e^{-2 \alpha h_{1}} \bar{Q}_{2}+e^{-2 \alpha h_{1}} \bar{R}_{11}-e^{-2 \alpha h_{1}} \bar{Q}_{1}-e^{-2 \alpha \bar{h}} \bar{S}_{1} \\
& -e^{-2 \alpha h_{1}} \bar{S}_{3}, \\
\Phi_{44}= & e^{-2 \alpha h_{1}} \bar{R}_{22}-e^{-2 \alpha \bar{h}} \bar{R}_{11}-e^{-2 \alpha \bar{h}} \bar{S}_{1}-e^{-2 \alpha h_{2}} \bar{S}_{2}, \\
\Phi_{55}= & -e^{-2 \alpha \bar{h}} \bar{R}_{22}-e^{-2 \alpha h_{2}} \bar{S}_{2}, \\
\Phi_{66}= & h_{1}^{2} \bar{S}_{3}+\delta^{2} \bar{S}_{2}+\delta^{2} \bar{S}_{1}+P^{-1} P^{-1}-2 P^{-1}, \\
\Phi_{13}= & e^{-2 \alpha h_{1}} \bar{S}_{3}, \\
\Phi_{23}= & e^{-2 \alpha \bar{h}}\left(\bar{S}_{1}-\bar{M}_{1}\right), \\
\Phi_{24}= & e^{-2 \alpha \bar{h}}\left(\bar{S}_{1}-\bar{M}_{1}^{T}\right), \\
\Phi_{34}= & e^{-2 \alpha h_{1}} \bar{R}_{12}+e^{-2 \alpha \bar{h}} \bar{M}_{1}^{T}, \\
\Phi_{45}= & -e^{-2 \alpha \bar{h}} \bar{R}_{12}+e^{-2 \alpha h_{2}} \bar{S}_{2} .
\end{aligned}
$$

If we pre- and postmultiply $\Phi_{1}$ by $\operatorname{diag}\{P, P, P, P, P, P\}$ and let

$$
K=Y P^{-1} \text {, }
$$

then the condition $\Phi_{1}<0$ is equivalent to condition (11) by using Schur Complement Lemma.

(ii) Assume now that $\bar{h} \leq h(t) \leq h_{2}$. Applications of Jensen's inequality [19] and Lemma 5 yield

$$
\begin{aligned}
& -\delta \int_{t-h_{2}}^{t-\bar{h}} \dot{x}^{T}(s) \bar{S}_{2} \dot{x}(s) d s \\
& =-\delta\left[\int_{t-h_{2}}^{t-h(t)} \dot{x}^{T}(s) \bar{S}_{2} \dot{x}(s) d s\right. \\
& \left.+\int_{t-h(t)}^{t-\bar{h}} \dot{x}^{T}(s) \bar{S}_{2} \dot{x}(s) d s\right] \\
& \leq-\frac{\delta}{h_{2}-h(t)}\left(\int_{t-h_{2}}^{t-h(t)} \dot{x}(s) d s\right)^{T} \\
& \cdot \bar{S}_{2}\left(\int_{t-h_{2}}^{t-h(t)} \dot{x}(s) d s\right)
\end{aligned}
$$

$$
\begin{aligned}
& -\frac{\delta}{h(t)-\bar{h}}\left(\int_{t-h(t)}^{t-\bar{h}} \dot{x}(s) d s\right)^{T} \bar{S}_{2}\left(\int_{t-h(t)}^{t-\bar{h}} \dot{x}(s) d s\right) \\
& \leq-\left[\begin{array}{c}
x(t-h(t))-x\left(t-h_{2}\right) \\
x(t-\bar{h})-x(t-h(t))
\end{array}\right]^{T} \\
& \cdot\left[\begin{array}{cc}
\bar{S}_{2} & \bar{M}_{2} \\
* & \bar{S}_{2}
\end{array}\right]\left[\begin{array}{c}
x(t-h(t))-x\left(t-h_{2}\right) \\
x(t-\bar{h})-x(t-h(t))
\end{array}\right],
\end{aligned}
$$

and hence

$$
\begin{aligned}
- & \delta e^{-2 \alpha h_{2}} \int_{t-h_{2}}^{t-\bar{h}} \dot{x}^{T}(s) \bar{S}_{2} \dot{x}(s) d s \\
& \leq-\left[\begin{array}{c}
x(t-h(t))-x\left(t-h_{2}\right) \\
x(t-\bar{h})-x(t-h(t))
\end{array}\right]^{T} \\
& \cdot\left[\begin{array}{cc}
e^{-2 \alpha h_{2}} \bar{S}_{2} & e^{-2 \alpha h_{2}} \bar{M}_{2} \\
* & e^{-2 \alpha h_{2}} \bar{S}_{2}
\end{array}\right]\left[\begin{array}{c}
x(t-h(t))-x\left(t-h_{2}\right) \\
x(t-\bar{h})-x(t-h(t))
\end{array}\right] .
\end{aligned}
$$

Note that when $h(t)=\bar{h}$ or $h(t)=h_{2}$, one can obtain $\int_{t-\bar{h}}^{t-h(t)} \dot{x}(s) d s=0$ or $\int_{t-h(t)}^{t-h_{2}} \dot{x}(s) d s=0$, respectively. Therefore, inequality (36) is satisfied.

Using Jensen's inequality [19], we deduce that

$$
\begin{gathered}
-\delta e^{-2 \alpha \bar{h}} \int_{t-\bar{h}}^{t-h_{1}} \dot{x}^{T}(s) \bar{S}_{1} \dot{x}(s) d s \\
\leq-\left[x\left(t-h_{1}\right)-x(t-\bar{h})\right]^{T} \\
\cdot e^{-2 \alpha \bar{h}} \bar{S}_{1}\left[x\left(t-h_{1}\right)-x(t-\bar{h})\right] .
\end{gathered}
$$

Combining (19)-(25), (28), and (30)-(37), we obtain

$$
\dot{V}\left(x_{t}\right)+2 \alpha V\left(x_{t}\right) \leq \xi^{T}(t) \Phi_{2} \xi(t)+6 \beta^{2}(t),
$$

where

$$
\begin{aligned}
& \Phi_{2} \\
& =\left[\begin{array}{cccccc}
\Phi_{11} & P^{-1} D & \Phi_{13} & 0 & 0 & (A+B K)^{T} P^{-1} \\
* & \bar{\Phi}_{22} & 0 & \bar{\Phi}_{24} & \bar{\Phi}_{25} & D^{T} P^{-1} \\
* & * & \Phi_{33} & \bar{\Phi}_{34} & 0 & 0 \\
* & * & * & \Phi_{44} & \bar{\Phi}_{45} & 0 \\
* & * & * & * & \Phi_{55} & 0 \\
* & * & * & * & * & \Phi_{66}
\end{array}\right], \\
& \bar{\Phi}_{22} \\
& =-(1-\mu) e^{-2 \alpha h_{2}} \bar{Q}_{2}+e^{-2 \alpha h_{2}} \bar{M}_{2}+e^{-2 \alpha h_{2}} \bar{M}_{2}^{T} \\
& -2 e^{-2 \alpha h_{2}} \bar{S}_{2}+6 b^{2} I,
\end{aligned}
$$




$$
\begin{aligned}
& \bar{\Phi}_{24}=e^{-2 \alpha h_{2}}\left(\bar{S}_{2}-\bar{M}_{2}\right), \\
& \bar{\Phi}_{25}=e^{-2 \alpha h_{2}}\left(\bar{S}_{2}-\bar{M}_{2}^{T}\right), \\
& \bar{\Phi}_{34}=e^{-2 \alpha h_{1}} \bar{R}_{12}+e^{-2 \alpha \bar{h}} \bar{S}_{1}, \\
& \bar{\Phi}_{45}=-e^{-2 \alpha \bar{h}} \bar{R}_{12}+e^{-2 \alpha h_{2}} \bar{M}_{2}^{T} .
\end{aligned}
$$

Pre- and postmultiplying $\Phi_{2}$ by $\operatorname{diag}\{P, P, P, P, P, P\}$ and letting $K=Y P^{-1}$, the condition $\Phi_{2}<0$ is equivalent to condition (12) by using Schur Complement Lemma.

From the above discussion, if conditions (11)-(13) are satisfied, then

$$
\dot{V}\left(x_{t}\right)+2 \alpha V\left(x_{t}\right) \leq 6 \beta^{2}(t), \quad t \geq 0 .
$$

By virtue of (40) and the definition of $V(x(t))$,

$$
\begin{aligned}
\lambda\|x(t, \phi)\|^{2} \leq V(x(t)) \\
\quad \leq e^{-2 \alpha t}\left(V(x(0))+6 \int_{0}^{t} \beta^{2}(s) e^{\int_{0}^{s} 2 \alpha d \tau} d s\right) \\
\quad \leq e^{-2 \alpha t}\left(\Gamma\|\phi\|^{2}+6 \int_{0}^{+\infty} \beta^{2}(s) e^{2 \alpha s} d s\right)=\Lambda e^{-2 \alpha t} .
\end{aligned}
$$

Hence, we have

$$
\|x(t, \phi)\| \leq \sqrt{\frac{\Lambda}{\lambda}} e^{-\alpha t}, \quad t \geq 0
$$

which implies that the closed-loop system is $\alpha$-stable. The proof is completed.

If there is no perturbation in system (1), that is, $f(t, x(t), x(t-h(t)))=0$, then system (1) reduces to

$$
\begin{aligned}
& \dot{x}(t)=A x(t)+D x(t-h(t))+B u(t), \quad t \geq 0, \\
& x(t)=\phi(t), \quad t \in\left[-h_{2}, 0\right] .
\end{aligned}
$$

Application of Theorem 6 yields the following result.

Corollary 7. Assume that $\alpha>0$ and condition (2) is satisfied. If there exist matrices $P>0, Q_{1}>0, Q_{2}>0, S_{i}>0(i=$ $1,2,3),\left[\begin{array}{cc}R_{11} & R_{12} \\ * & R_{22}\end{array}\right]>0, M_{1}, M_{2}$, and $Y$ such that

$$
\Xi_{1}=\left[\begin{array}{cccccc}
\Xi_{11} & D P & \Xi_{13} & 0 & 0 & P A^{T}+Y^{T} B^{T} \\
* & \Xi_{22} & \Xi_{23} & \Xi_{24} & 0 & P D^{T} \\
* & * & \Xi_{33} & \Xi_{34} & 0 & 0 \\
* & * & * & \Xi_{44} & \Xi_{45} & 0 \\
* & * & * & * & \Xi_{55} & 0 \\
* & * & * & * & * & \Xi_{66}
\end{array}\right]<0,
$$

$$
\Xi_{2}=\left[\begin{array}{cccccc}
\Xi_{11} & D P & \Xi_{13} & 0 & 0 & P A^{T}+Y^{T} B^{T} \\
* & \bar{\Xi}_{22} & 0 & \bar{\Xi}_{24} & \bar{\Xi}_{25} & P D^{T} \\
* & * & \Xi_{33} & \bar{\Xi}_{34} & 0 & 0 \\
* & * & * & \Xi_{44} & \bar{\Xi}_{45} & 0 \\
* & * & * & * & \Xi_{55} & 0 \\
* & * & * & * & * & \Xi_{66}
\end{array}\right]<0,
$$$$
\left[\begin{array}{cc}
S_{i} & M_{i} \\
* & S_{i}
\end{array}\right] \geq 0 \quad(i=1,2),
$$

where

$$
\begin{aligned}
& \Xi_{11}=A P+P A^{T}+B Y+Y^{T} B^{T}+2 \alpha P+Q_{1} \\
& -e^{-2 \alpha h_{1}} S_{3} \\
& \Xi_{22}=-(1-\mu) e^{-2 \alpha h_{2}} Q_{2}+e^{-2 \alpha \bar{h}} M_{1}+e^{-2 \alpha \bar{h}} M_{1}^{T} \\
& -2 e^{-2 \alpha \bar{h}} S_{1} \text {, } \\
& \Xi_{33}=e^{-2 \alpha h_{1}} Q_{2}+e^{-2 \alpha h_{1}} R_{11}-e^{-2 \alpha h_{1}} Q_{1}-e^{-2 \alpha \bar{h}} S_{1} \\
& -e^{-2 \alpha h_{1}} S_{3} \text {, } \\
& \Xi_{44}=e^{-2 \alpha h_{1}} R_{22}-e^{-2 \alpha \bar{h}} R_{11}-e^{-2 \alpha \bar{h}} S_{1}-e^{-2 \alpha h_{2}} S_{2} \text {, } \\
& \Xi_{55}=-e^{-2 \alpha \bar{h}} R_{22}-e^{-2 \alpha h_{2}} S_{2} \text {, } \\
& \Xi_{66}=h_{1}^{2} S_{3}+\delta^{2} S_{2}+\delta^{2} S_{1}-2 P, \\
& \Xi_{13}=e^{-2 \alpha h_{1}} S_{3} \text {, } \\
& \Xi_{23}=e^{-2 \alpha \bar{h}}\left(S_{1}-M_{1}\right) \text {, } \\
& \Xi_{24}=e^{-2 \alpha \bar{h}}\left(S_{1}-M_{1}^{T}\right) \text {, } \\
& \Xi_{34}=e^{-2 \alpha h_{1}} R_{12}+e^{-2 \alpha \bar{h}} M_{1}^{T} \text {, } \\
& \Xi_{45}=-e^{-2 \alpha \bar{h}} R_{12}+e^{-2 \alpha h_{2}} S_{2} \text {, } \\
& \bar{\Xi}_{22}=-(1-\mu) e^{-2 \alpha h_{2}} Q_{2}+e^{-2 \alpha h_{2}} M_{2}+e^{-2 \alpha h_{2}} M_{2}^{T} \\
& -2 e^{-2 \alpha h_{2}} S_{2} \\
& \bar{\Xi}_{24}=e^{-2 \alpha h_{2}}\left(S_{2}-M_{2}\right), \\
& \bar{\Xi}_{25}=e^{-2 \alpha h_{2}}\left(S_{2}-M_{2}^{T}\right) \text {, } \\
& \bar{\Xi}_{34}=e^{-2 \alpha h_{1}} R_{12}+e^{-2 \alpha \bar{h}} S_{1} \text {, } \\
& \bar{\Xi}_{45}=-e^{-2 \alpha \bar{h}} R_{12}+e^{-2 \alpha h_{2}} M_{2}^{T} \text {, }
\end{aligned}
$$

then system (43) is robustly $\alpha$-stabilizable, the state feedback control $u(t)=Y P^{-1} x(t)$, and the solution $x(t, \phi)$ of the closedloop system satisfies

$$
\|x(t, \phi)\| \leq \sqrt{\frac{\Lambda}{\lambda}} e^{-\alpha t}=\sqrt{\frac{\Gamma}{\lambda}} e^{-\alpha t}\|\phi\|, \quad t \geq 0 .
$$




\section{Numerical Examples}

In this section, two numerical examples are given to illustrate the effectiveness of the results obtained in this paper.

Example 1. Consider the system with a nonlinear perturbation

$$
\begin{aligned}
\dot{x}(t)= & A x(t)+D x(t-h(t)) \\
& +f(t, x(t), x(t-h(t)))+B u(t), \\
x(t)= & {\left[\begin{array}{ll}
1 & 1
\end{array}\right]^{T}, \quad t \in[-0.4,0], }
\end{aligned}
$$

where

$$
\begin{aligned}
& A=\left[\begin{array}{cc}
1 & 0 \\
0 & -1
\end{array}\right], \\
& B=\left[\begin{array}{l}
0 \\
1
\end{array}\right], \\
& D=\left[\begin{array}{cc}
-2 & -0.1 \\
0 & 1.1
\end{array}\right],
\end{aligned}
$$

$h(t)=0.1+0.3 \sin ^{2}(5 t / 3)$, and the nonlinear perturbation satisfies

$$
\begin{aligned}
& \|f(t, x(t), x(t-h(t)))\| \\
& \quad \leq 0.1\|x(t)\|+0.1\|x(t-h(t))\|+e^{-0.66 t} .
\end{aligned}
$$

Note that $h_{1}=0.1, h_{2}=0.4, \mu=0.5$, and $\beta(t)=e^{-0.66 t}$. It is not difficult to check that $A$ and $A+D$ are Hurwitz unstable.

Let $\alpha=0.16$. Then $\int_{0}^{+\infty} \beta^{2}(s) e^{2 \alpha s} d s=\int_{0}^{+\infty} e^{-s} d s=1<$ $+\infty$. Using the LMI Toolbox in MATLAB, LMIs (11)-(13) in Theorem 6 are satisfied with

$$
\begin{aligned}
P & =\left[\begin{array}{cc}
4.4365 & -0.1306 \\
-0.1306 & 4.9389
\end{array}\right], \\
Q_{1} & =\left[\begin{array}{ll}
0.1510 & 0.4068 \\
0.4068 & 3.4481
\end{array}\right], \\
Q_{2} & =\left[\begin{array}{ll}
0.0115 & 0.0066 \\
0.0066 & 0.2019
\end{array}\right], \\
S_{1} & =\left[\begin{array}{ll}
62.2011 & -5.1166 \\
-5.1166 & 52.7981
\end{array}\right], \\
S_{2} & =\left[\begin{array}{ll}
36.2972 & -1.8408 \\
-1.8408 & 31.9872
\end{array}\right], \\
S_{3} & =\left[\begin{array}{ll}
71.6879 & -4.4063 \\
-4.4063 & 40.8855
\end{array}\right], \\
R_{11} & =\left[\begin{array}{ll}
12.6433 & -1.6878 \\
-1.6878 & 21.3645
\end{array}\right],
\end{aligned}
$$

$$
\begin{aligned}
R_{12} & =\left[\begin{array}{cc}
-12.6769 & 2.2144 \\
2.2144 & -18.8952
\end{array}\right], \\
R_{22} & =\left[\begin{array}{cc}
13.2723 & -1.3613 \\
-1.3613 & 21.8765
\end{array}\right], \\
M_{1} & =\left[\begin{array}{ll}
11.3018 & -6.7305 \\
-6.7305 & 20.9430
\end{array}\right], \\
M_{2} & =\left[\begin{array}{ll}
-14.5051 & -2.3510 \\
-2.3510 & 0.3503
\end{array}\right], \\
Y & =\left[\begin{array}{ll}
0.2410 & -6.8850
\end{array}\right] .
\end{aligned}
$$

Furthermore, the solution $x(t, \phi)$ of the system satisfies

$$
\|x(t, \phi)\| \leq 6.1258 e^{-0.16 t}, \quad t \geq 0,
$$

and the stabilizing feedback control

$$
u(t)=\left[\begin{array}{ll}
0.0133 & -1.3937
\end{array}\right] x(t), \quad t \geq 0 .
$$

Observe that the results reported in $[3,8,16]$ cannot be applied to (47) since the nonlinear perturbation is related with the term $\beta(t)=e^{-0.66 t}$.

Example 2. Consider a linear system with an interval timevarying delay

$$
\begin{aligned}
& \dot{x}(t)=A x(t)+D x(t-h(t))+B u(t), \quad t \geq 0, \\
& x(t)=\phi(t), \quad \forall t \in\left[-h_{2}, 0\right],
\end{aligned}
$$

where

$$
\begin{aligned}
& A=\left[\begin{array}{ll}
0 & 0 \\
0 & 1
\end{array}\right], \\
& B=\left[\begin{array}{l}
0 \\
1
\end{array}\right], \\
& D=\left[\begin{array}{ll}
-1 & -1 \\
0 & -0.9
\end{array}\right],
\end{aligned}
$$

and $h(t)=0.5+1.28 \sin ^{2}(25 t / 64)$. Note that $h_{1}=0.5, h_{2}=$ 1.78 , and $\mu=0.5$. It is easy to check that $A$ and $A+D$ are Hurwitz unstable. Given $\alpha=0.01$, using the LMI Toolbox in MATLAB, LMIs (44) in Corollary 7 are satisfied with

$$
\begin{aligned}
P & =\left[\begin{array}{cc}
84.8721 & -32.8554 \\
-32.8554 & 18.7095
\end{array}\right], \\
Q_{1} & =\left[\begin{array}{cc}
0.0864 & -0.0424 \\
-0.0424 & 11.7837
\end{array}\right], \\
Q_{2} & =\left[\begin{array}{ll}
0.2099 & 0.2035 \\
0.2035 & 11.4665
\end{array}\right],
\end{aligned}
$$




$$
\begin{aligned}
S_{1} & =\left[\begin{array}{cc}
109.3732 & -42.3701 \\
-42.3701 & 16.5117
\end{array}\right], \\
S_{2} & =\left[\begin{array}{cc}
102.7380 & -39.7555 \\
-39.7555 & 15.5155
\end{array}\right], \\
S_{3} & =\left[\begin{array}{cc}
124.7698 & -48.3825 \\
-48.3825 & 18.8240
\end{array}\right], \\
R_{11} & =\left[\begin{array}{cc}
32.4350 & -12.8325 \\
-12.8325 & 5.1838
\end{array}\right], \\
R_{12} & =\left[\begin{array}{cc}
-2.3196 & 0.9331 \\
0.9331 & -0.3969
\end{array}\right], \\
R_{22} & =\left[\begin{array}{cc}
54.1560 & -21.0105 \\
-21.0105 & 8.2532
\end{array}\right], \\
M_{1} & =\left[\begin{array}{cc}
-22.9733 & 9.4881 \\
9.4881 & -3.9352
\end{array}\right], \\
M_{2} & =\left[\begin{array}{cc}
-53.6877 & 20.8764 \\
20.8764 & -8.2092
\end{array}\right], \\
Y & =\left[\begin{array}{cc}
23.8997 & -27.0789
\end{array}\right] .
\end{aligned}
$$

Moreover, the solution $x(t, \phi)$ of the system satisfies

$$
\|x(t, \phi)\| \leq 9.3399 e^{-0.01 t}\|\phi\|, \quad t \geq 0
$$

and the stabilizing feedback control

$$
u(t)=\left[\begin{array}{ll}
-0.8704 & -2.9758
\end{array}\right] x(t), \quad t \geq 0 .
$$

Figure 1 shows the trajectories of $x_{1}(t)$ and $x_{2}(t)$ of the open-loop system with the initial condition $\phi(t)=\left[\begin{array}{ll}30 & 10\end{array}\right]^{T}$, $t \in[-1.78,0]$. Figure 2 shows the trajectories of $x_{1}(t)$ and $x_{2}(t)$ of the closed-loop system with the state feedback $u(t)=$ $\left[\begin{array}{ll}-0.8704 & -2.9758\end{array}\right] x(t)$ and the initial condition $\phi(t)=$ $\left[\begin{array}{ll}30 & 10\end{array}\right]^{T}, t \in[-1.78,0]$.

Letting the lower and upper bounds of the time delay be the same as in Zhang et al. [12], our results also ensure exponential stability with an $\alpha$-convergence rate as given in Table 1. Note that Zhang et al. [12] discussed asymptotic stability, whereas the controller derived in this paper provides exponential stability for the closed-loop system. Furthermore, the maximum bound for $\alpha$ is better than Thuan et al. [16] by letting $h_{1}$ and $h_{2}$ be the same as in [16]. For selected $h_{1}$ and $\mu=0.5$, using Corollary 7 , one can easily observe that the maximum allowable delay bounds for $h_{2}$ are better than those reported in the papers by Zhang et al. [12] and Thuan et al. [16].

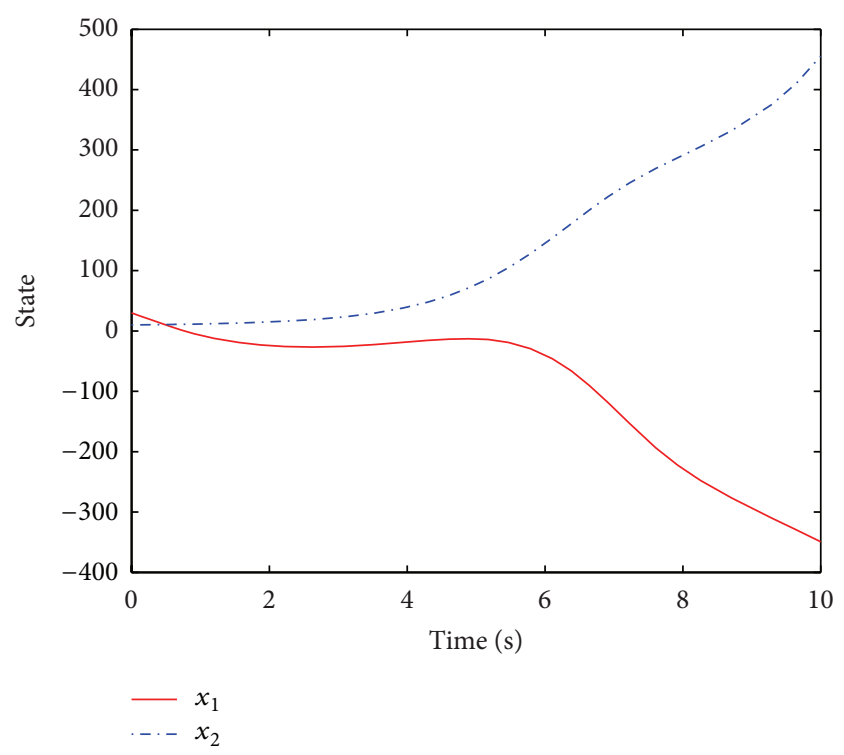

Figure 1: Open-loop trajectories of $x_{1}(t)$ and $x_{2}(t)$.

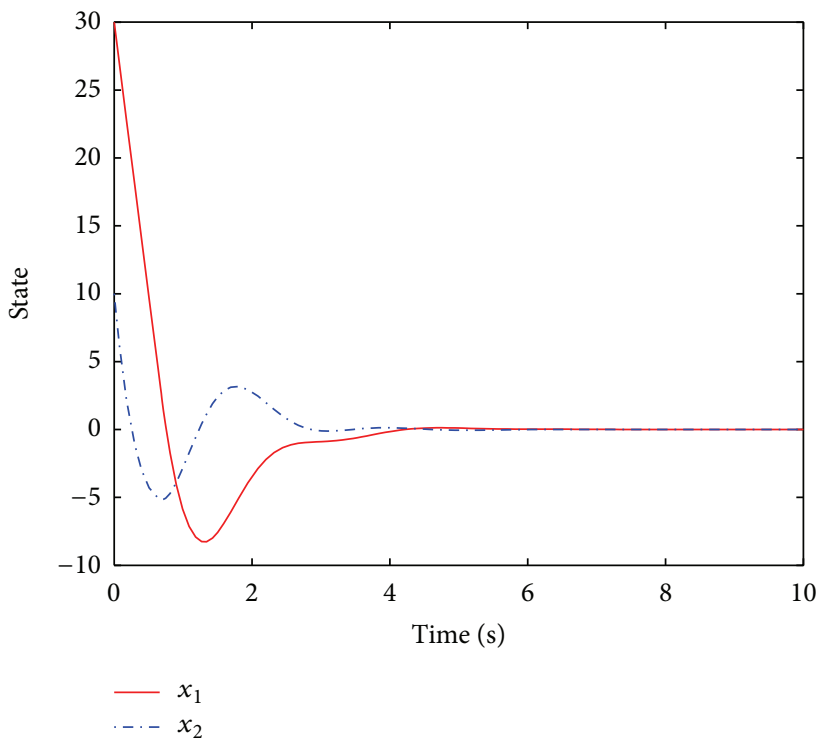

FIGURE 2: Closed-loop trajectories of $x_{1}(t)$ and $x_{2}(t)$.

\section{Conclusions}

In this paper, exponential stabilization of a class of timevarying delay systems with nonlinear perturbations has been investigated. By using the delay decomposition approach and constructing a novel Lyapunov functional, some new delay-dependent stabilization criteria are obtained in order to ensure closed-loop stability of the system with any prescribed $\alpha$-convergence rate. Numerical examples are given to illustrate that the results obtained are much less conservative than some existing results in the literature. Exponential stabilization of impulsive switched delay systems with nonlinear perturbations will be further investigated in the future. 
TABLE 1: Admissible upper bound $h_{2}$ and $K$ for given $h_{1}$ with $\mu=0.5$.

\begin{tabular}{lccc}
\hline Method & $h_{1}$ & $h_{2}$ & $K$ \\
\hline Zhang et al. [12] & 0.5 & 0.967 & {$\left[\begin{array}{ll}-0.5290 & -2.7166\end{array}\right]$} \\
Thuan et al. [16] $(\alpha=0.314)$ & 0.5 & 0.967 & {$\left[\begin{array}{ll}-0.0967 & -1.8338\end{array}\right]$} \\
Corollary 7 $(\alpha=0.353)$ & 0.5 & 0.967 & {$\left[\begin{array}{ll}-0.1662 & -2.0605\end{array}\right]$} \\
Thuan et al. [16] $(\alpha=0.01)$ & 0.5 & 1.66 & {$\left[\begin{array}{ll}-0.8765 & -2.9873\end{array}\right]$} \\
Corollary 7 $(\alpha=0.01)$ & 0.5 & 1.78 & {$\left[\begin{array}{ll}-0.8704 & -2.9758\end{array}\right]$} \\
Zhang et al. [12] & 1.0 & 1.114 & {$\left[\begin{array}{ll}-0.3096 & -2.1894\end{array}\right]$} \\
Thuan et al. $[16](\alpha=0.225)$ & 1.0 & 1.114 & {$\left[\begin{array}{ll}-0.6344 & -2.9059\end{array}\right]$} \\
Corollary 7 $(\alpha=0.236)$ & 1.0 & 1.114 & {$\left[\begin{array}{ll}-0.6714 & -2.9317\end{array}\right]$} \\
Thuan et al. [16] $(\alpha=0.01)$ & 1.0 & 1.672 & {$\left[\begin{array}{ll}-0.9023 & -2.9983\end{array}\right]$} \\
Corollary 7 $(\alpha=0.01)$ & 1.0 & 1.765 & {$\left[\begin{array}{lll}-0.8701 & -2.9712\end{array}\right]$} \\
\hline
\end{tabular}

\section{Conflict of Interests}

The authors declare that they have no conflict of interests.

\section{Acknowledgments}

The authors express their sincere gratitude to the editors and three anonymous referees for their constructive comments and suggestions that helped to improve the presentation of the results and accentuate important details. This work was supported by the Natural Science Foundation of Shandong Province under Grant no. JQ201119 and the National Natural Science Foundation of China under Grant nos. 61174217, 61374074 , and 61473133 .

\section{References}

[1] J. K. Hale and S. M. V. Lunel, Introduction to Functional Differential Equations, Springer, New York, NY, USA, 1993.

[2] M. Malek-Zavarei and M. Jamshidi, Time-Delay Systems: Analysis, Optimization and Applications, North-Holland, Amsterdam, The Netherklands, 1987.

[3] Q.-L. Han, "Robust stability for a class of linear systems with time-varying delay and nonlinear perturbations," Computers \& Mathematics with Applications, vol. 47, no. 8-9, pp. 1201-1209, 2004.

[4] F. Qiu, B. T. Cui, and Y. Ji, "Further results on robust stability of neutral system with mixed time-varying delays and nonlinear perturbations," Nonlinear Analysis. Real World Applications, vol. 11, no. 2, pp. 895-906, 2010.

[5] R. Rakkiyappan, P. Balasubramaniam, and R. Krishnasamy, "Delay dependent stability analysis of neutral systems with mixed time-varying delays and nonlinear perturbations," Journal of Computational and Applied Mathematics, vol. 235, no. 8, pp. 2147-2156, 2011.

[6] W. Wang, S. K. Nguang, S. Zhong, and F. Liu, "Novel delaydependent stability criterion for time-varying delay systems with parameter uncertainties and nonlinear perturbations," Information Sciences, vol. 281, pp. 321-333, 2014.

[7] L. D. Guo, H. Gu, J. Xing, and X. Q. He, "Asymptotic and exponential stability of uncertain system with interval delay,"
Applied Mathematics and Computation, vol. 218, no. 19, pp. 9997-10006, 2012.

[8] W. Zhang, X.-S. Cai, and Z.-Z. Han, "Robust stability criteria for systems with interval time-varying delay and nonlinear perturbations," Journal of Computational and Applied Mathematics, vol. 234, no. 1, pp. 174-180, 2010.

[9] M. Li and G. D. Hu, "Delay-dependent robust stabilization for a class of neutral systems with nonlinear perturbations," Journal of Control Theory and Applications, vol. 5, no. 4, pp. 409-414, 2007.

[10] T. Li, L. Guo, and Y. M. Zhang, "Delay-range-dependent robust stability and stabilization for uncertain systems with timevarying delay," International Journal of Robust and Nonlinear Control, vol. 18, no. 13, pp. 1372-1387, 2008.

[11] P. G. Wang and X. Liu, “ $\phi_{0}$-stability of hybrid impulsive dynamic systems on time scales," Journal of Mathematical Analysis and Applications, vol. 334, no. 2, pp. 1220-1231, 2007.

[12] J. Zhang, Y. Xia, P. Shi, and M. S. Mahmoud, "New results on stability and stabilisation of systems with interval time-varying delay," IET Control Theory \& Applications, vol. 5, no. 3, pp. 429436, 2011.

[13] P.-L. Liu, "State feedback stabilization of time-varying delay uncertain systems: a delay decomposition approach," Linear Algebra and its Applications, vol. 438, no. 5, pp. 2188-2209, 2013.

[14] L. V. Hien and V. N. Phat, "Exponential stability and stabilization of a class of uncertain linear time-delay systems," Journal of the Franklin Institute, vol. 346, no. 6, pp. 611-625, 2009.

[15] J. Cheng, H. Zhu, S. M. Zhong, and G. H. Li, "Novel delay-dependent robust stability criteria for neutral systems with mixed time-varying delays and nonlinear perturbations," Applied Mathematics and Computation, vol. 219, no. 14, pp. 77417753, 2013.

[16] M. V. Thuan, V. N. Phat, T. Fernando, and H. Trinh, "Exponential stabilization of time-varying delay systems with nonlinear perturbations," IMA Journal of Mathematical Control and Information, vol. 31, no. 4, pp. 441-464, 2014.

[17] Y. G. Sun, L. Wang, and G. M. Xie, "Exponential stability of switched systems with interval time-varying delay," IET Control Theory \& Applications, vol. 3, no. 8, pp. 1033-1040, 2009.

[18] Y. G. Sun and J. Qi, "Note on exponential stability of certain nonlinear differential systems with time-varying delays," Applied Mathematics Letters, vol. 25, no. 12, pp. 2240-2245, 2012.

[19] K. Gu, "An integral inequality in the stability problem of time delay systems," in Proceedings of the 39th IEEE Conference on Decision Control, pp. 2805-2810, 2000.

[20] P. Park, J. W. Ko, and C. Jeong, "Reciprocally convex approach to stability of systems with time-varying delays," Automatica, vol. 47, no. 1, pp. 235-238, 2011.

[21] Y. J. Zhang, D. Yue, and E. G. Tian, "New stability criteria of neural networks with interval time-varying delay: a piecewise delay method," Applied Mathematics and Computation, vol. 208, no. 1, pp. 249-259, 2009.

[22] A. Seuret and F. Gouaisbaut, "Wirtinger-based integral inequality: application to time-delay systems," Automatica, vol. 49, no. 9, pp. 2860-2866, 2013.

[23] O. M. Kwon, J. H. Park, and S. M. Lee, "On robust stability criterion for dynamic systems with time-varying delays and nonlinear perturbations," Applied Mathematics and Computation, vol. 203, no. 2, pp. 937-942, 2008. 
[24] Y. Liu, S. M. Lee, O. M. Kwon, and J. H. Park, "Delay-dependent exponential stability criteria for neutral systems with interval time-varying delays and nonlinear perturbations," Journal of the Franklin Institute, vol. 350, no. 10, pp. 3313-3327, 2013.

[25] K. Zhou and P. P. Khargonekar, "Robust stabilization of linear systems with norm-bounded time-varying uncertainty," Systems \& Control Letters, vol. 10, no. 1, pp. 17-20, 1988. 


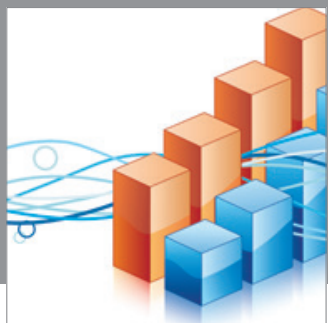

Advances in

Operations Research

mansans

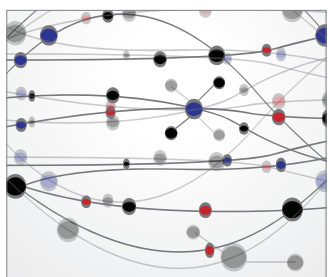

The Scientific World Journal
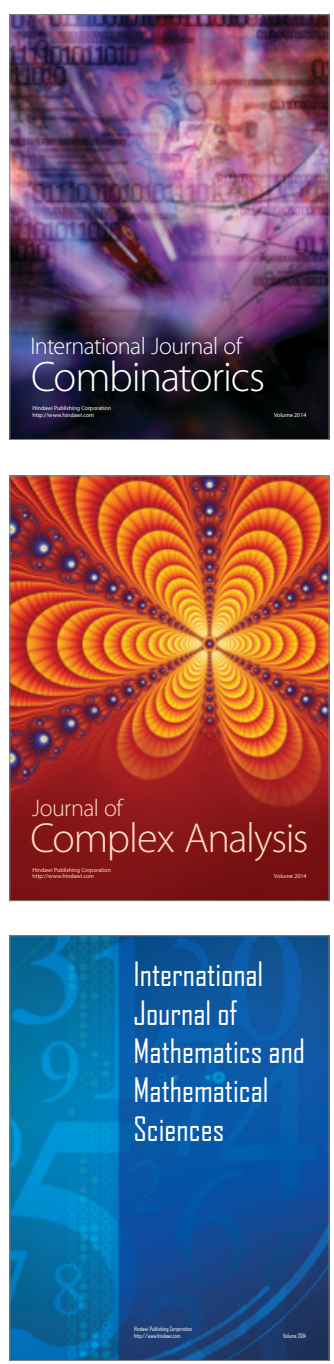
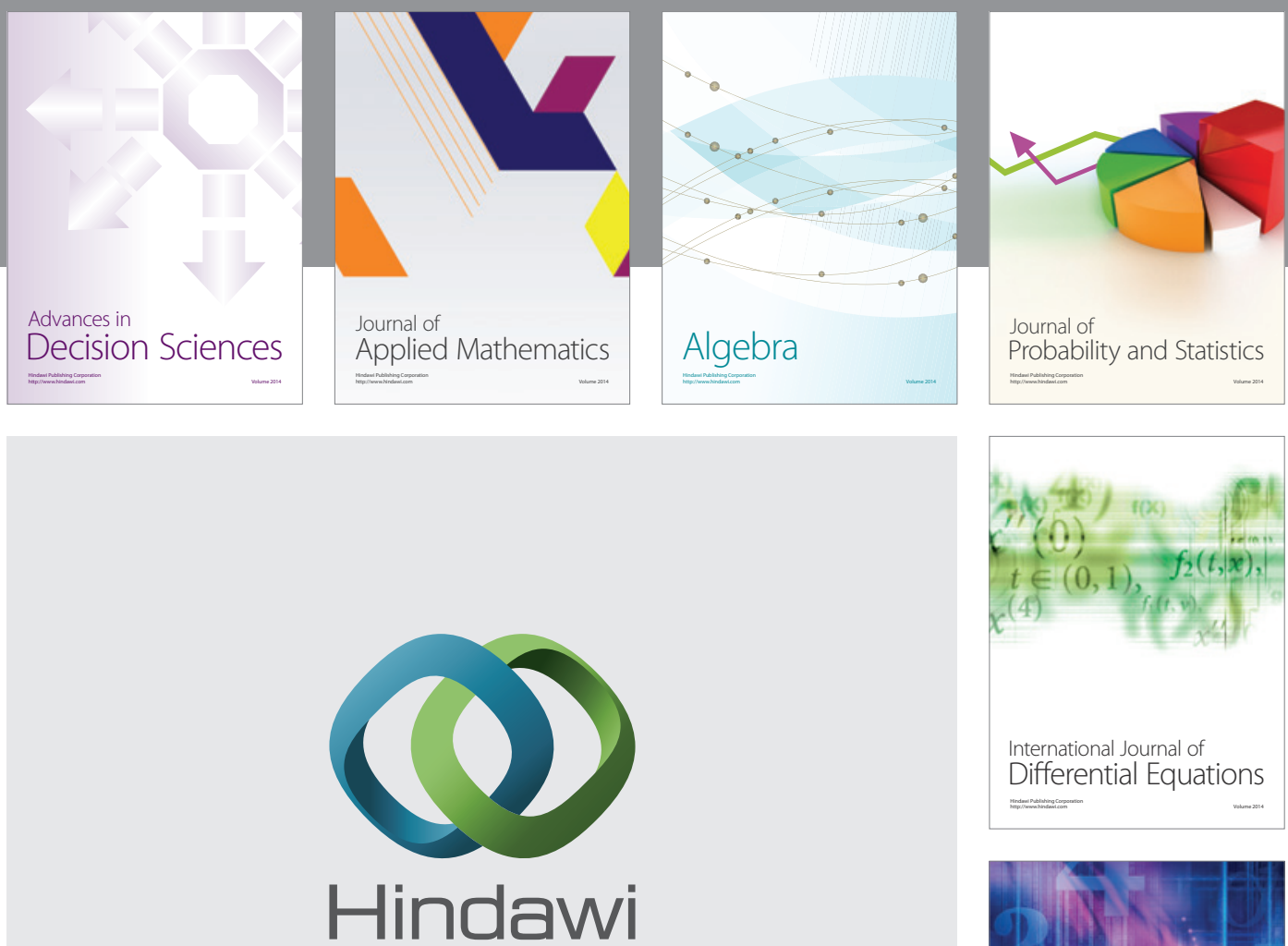

Submit your manuscripts at http://www.hindawi.com
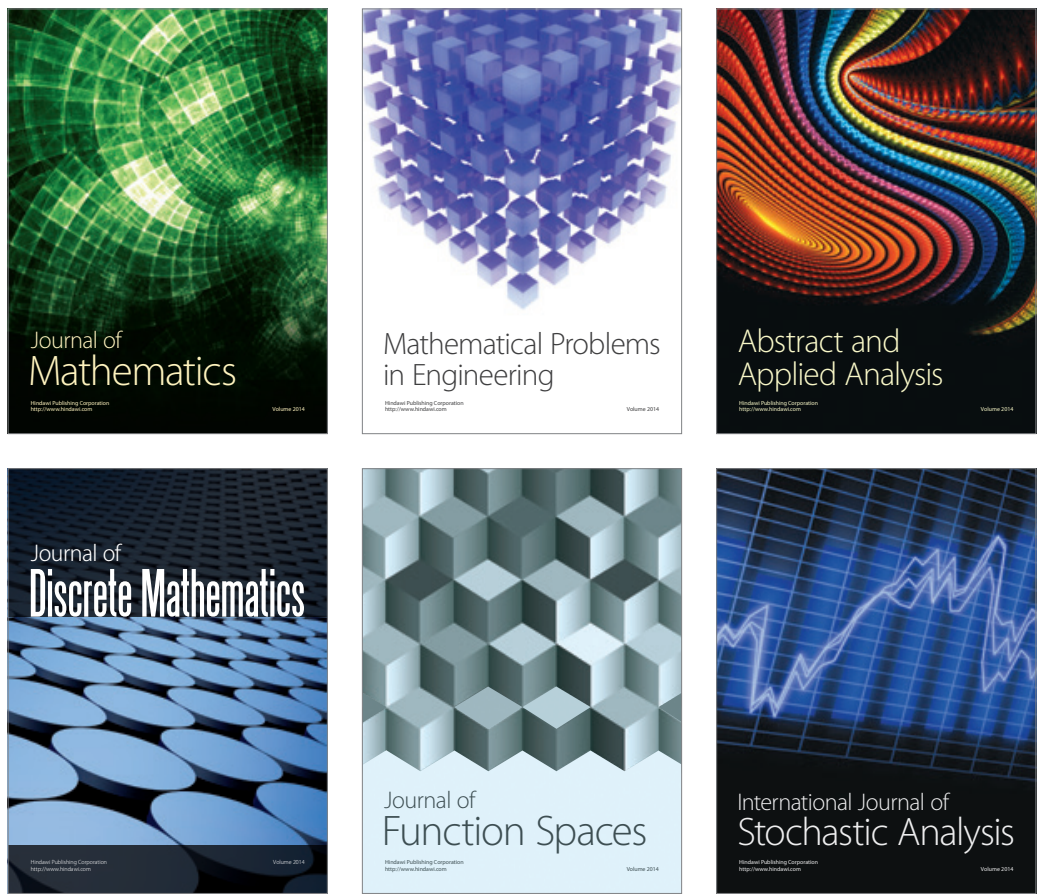

Journal of

Function Spaces

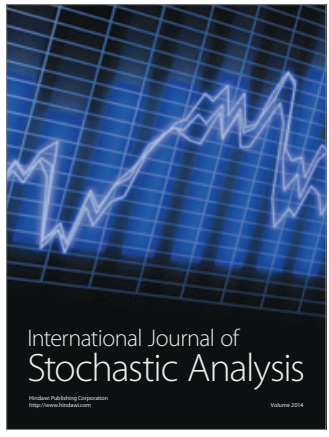

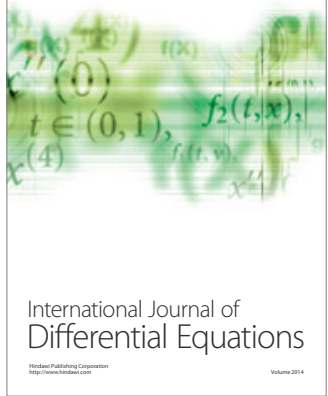
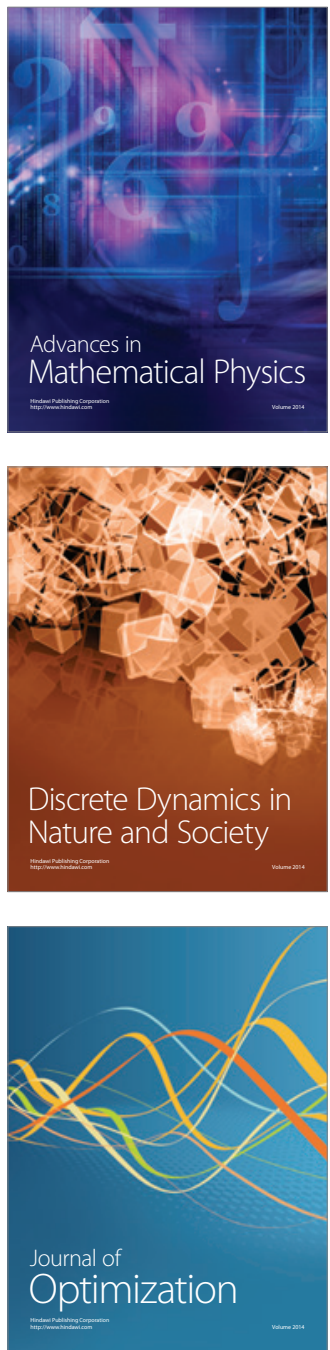